\title{
SOLAR SYSTEM SONIFICATION: EXPLORING EARTH AND ITS NEIGHBORS THROUGH SOUND
}

\author{
Brianna J. Tomlinson ${ }^{1}$, R. Michael Winters ${ }^{2}$, Christopher Latina ${ }^{2}$, \\ Smruthi Bhat ${ }^{3}$, Milap Rane ${ }^{2}$, Bruce N. Walker ${ }^{1,4}$ \\ School of Interactive Computing ${ }^{1}$, \\ Georgia Tech Center for Music Technology (GTCMT) ${ }^{2}$, \\ School of Computer Science ${ }^{3}$, School of Psychology ${ }^{4}$ \\ Georgia Institute of Technology, Atlanta, USA \\ btomlin@gatech.edu, mikewintersegatech.edu, chrisrlatinalgmail.com, \\ smruthib@gatech.edu, mrane3@gatech.edu, bruce.walker@psych.gatech.edu
}

\begin{abstract}
Informal learning environments (ILEs) like museums incorporate multi-modal displays into their exhibits as a way to engage a wider group of visitors, often relying on tactile, audio, and visual means to accomplish this. Planetariums, however, represent one type of ILE where a single, highly visual presentation modality is used to entertain, inform, and engage a large group of users in a passive viewing experience. Recently, auditory displays have been used as a supplement or even an alternative to visual presentation of astronomy concepts, though there has been little evaluation of those displays. Here, we designed an auditory model of the solar system and created a planetarium show, which was later presented at a local science center. Attendees evaluated the performance on helpfulness, interest, pleasantness, understandability, and relatability of the sounds mappings. Overall, attendees rated the solar system and planetary details very highly, in addition to providing open-ended responses about their entire experience.
\end{abstract}

\section{INTRODUCTION}

Museums and other ILEs have explored multi-modal exhibits to increase engagement and prolong interaction for attendees [1, 2, 3] Multi-modal exhibit design provides additional methods for presenting content to visitors, enhancing the experience for everyone, and allowing greater access to those with impairments [4]. Larger movements in incorporating universal design have resulted in greater development and evaluation of accessibility in these learning environments [5]. Descriptive audio tours and other auditory displays can support shared experiences for larger groups of visitors [6] and provide exploration methods for those with vision impairment $[7,8,9]$. One example, the Aquarium Sonification created dynamic soundscapes through mapping fish characteristics and events within the tank, as a way to provide a unique experience for individual exhibits [3]. On the other hand, not all ILEs use multiple modalities in their presentations. As one example, planetarium shows are typically visual-only; or, if there is any

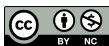

Thork is licensed under Creative Commons Attribution Non Commercial 4.0 International License. The full terms of the License are available at http://creativecommons.org/licenses/by-nc/4.0 audio it is largely supplementary.

This paper explores and expands the use of these auditory experiences in a planetarium show-a typically informal, passive learning experience that is predominantly visual. Leveraging the possibilities of spatial audio, a variety of quantitative information about each planet in our solar system was conveyed to an audience through sound (with some static visual anchors). Surveys collected during the show demonstrate that the show was interesting, understandable, relatable and helpful, even to a sample audience without visual impairments. The results hold promise for the creation of future shows that entertain and educate through listening.

\section{RELATED WORK}

Sonification, or the use of non-speech sound to present information, has been explored and used for a variety of situations and applications as a type of auditory display [10]. Even though there is some precedence for using auditory displays as a way to promote public interest in space and astronomy, there has not been extensive evaluation of these displays. Previous work has focused on making sounds that are already collected through (radio) telescopes and other instruments available to the public. Harger and Hyde, and others have broadcast live sounds from radio telescopes over the internet and FM radio stations [11, 12].

Some work has explored using sonification and audification (direct mapping of a dataset to sounds) to analyze data sets from space, such as Cosmic Microwave Background Radiation or the Search for Extra-Terrestrial Intelligence (SETI) [13]. Other recent work includes Landi et al.'s analysis of solar wind through audification of solar rotation data to explore carbon ionization [14]. Ballora created more musically-composed sonifications for an outreach film presented at the Smithsonian Air \& Space Museum, but did not evaluate their success in presenting the information to attendees [15].

Recently, Quinton et al. developed a model for representing characteristics of the Solar System [16]. Through an interview with a planetarium representative, they identified seven important concepts to include in their model (density, diameter, gravity, length of day, orbital period, temperature, and orbital velocity). They completed a small-scale evaluation with 12 users, where each participant was asked to identify characteristics for each planet when listening to the sonification. Though they gathered valuable 
feedback about their sonifications, this work was focused more on individual interpretation of the model without scaffolding their experience and knowledge and did not evaluate the work in an ecologically valid manner.

Exploring how to represent planetary data in a multi-modal experience and evaluating different characteristics of the design for the solar system model presents a novel, artistic, and interesting area to explore, which has the potential to reach into other applications in both informal learning spaces and formal learning activities.

\section{IDENTIFYING IMPORTANT CONCEPTS}

At the beginning of the development, we wanted to better understand which concepts were most important for teaching a comprehensive understanding about the solar system. To do this, we conducted semi-structured interviews with five teachers across different levels of science classes, including a planetarium instructor, a university professor who teaches intro level astronomy, and three additional teachers across elementary through high school. All of the teachers had at least 9 years of teaching experience, with a few having almost 30 years of experience teaching astronomy.

During the interviews, we asked about the types of spacerelated concepts they teach, some detailed examples of how they introduce the topics, and the types of misconceptions that confuse their students (or audience) the most. We used these interviews as a way to explore how to structure the introduction of the topics in the planetarium show and a way to identify which information people struggle with learning the most.

A common theme that arose from these interviews was the lack of prior knowledge most people have about astronomy: the teachers need to start their lessons by introducing everything from the ground up. Many of the teachers described comparing and contrasting features of the planets such as the gravitational strength, atmosphere and surface composition, and other details such as rings and moons. The planetarium instructor mentioned mixing different levels of detail for each of the topics, in case some individuals in her audience already know the basics. This is more of a concern for her than it is for the other teachers, who typically have more homogeneous groups of learners.

When asked about common misconceptions their students have, all of the teachers said that understanding the scale of the solar system (and space in general) was one of the hardest concepts to convey. Another common misconception dealt with understanding seasons (and their relation to the tilt of a planet) and the elliptical nature of orbits.

Using the interview responses from the teachers, we decided to focus our sonification on details related to the scale of the solar system, such as mass, temperature, and distance.

\section{SOUND DESIGN}

The process for designing the sounds for the show was focused around two "views" of the solar system. In the first view, the Solar System View, the planets' masses, lengths of years, lengths of days, and distances from the sun were compared to each other, first as if the listeners were on the sun, hearing them rotate around; and then as if traveling on a space-ship visiting each planet on the way. This view worked to address the misconception of size and scale identified through the interviews, and presented a baseline of information about the planets for everyone in the audience.
In the second view, the Planetary View, the desire was to provide an experience of what it would be like to be on a given planet's surface. In this view, each planet's type, number of moons, number of rings, gravitational force, and temperature range were conveyed. This view provided additional details about the features of the planets and allowed for comparisons between them.

For all of the cases, sound was spatialized using the planetarium's quadraphonic speaker system and Vector-Based Amplitude Panning (VBAP). This configuration allowed sources to rotate around the audience at variable speeds. For example, in the Solar System View, the sounds representing the planets were first distributed spatially, and rotated around the listener at a rate proportional to the orbital period of that planet around the sun. Two speed factors were introduced to make the planet's relative motions fast enough to be perceived by ear, one factor was applied to the terrestrial planets and one factor for the gas giants (which are much slower). For the Planetary View, a similar approach was taken for moons and rings, though without taking into account the specific orbital periods of individual moons. The choice of a quadraphonic speaker algorithm was guided by the affordances of the planetarium's audio system, which already included four equidistant speakers positioned in a ring around the audience area.

Prototyping and designing the sonification made use of $\mathrm{Su}$ perCollider [17, 18], an open-source computer music library commonly used in sonification [19]. Data for sonification were downloaded from NASA's Planetary Fact Sheet [20], and mapping principles were derived from principles of stream segregation and parameter nesting, as well as designing to support spatial audio $[21,22,23,24]$.

\subsection{Solar System View}

The Solar System View individually conveyed the mass of each planet, the length of year, the length of day, and the distance of each planet from the sun. To create the fundamental sound of each planet, brown noise was used, and a resonant filter was applied whose center frequency scaled proportionally to the mass of the planet. Following the polarity mappings of previous work [25], the mass of each planet was mapped inversely to pitch (i.e., sounds for larger planets had a lower pitch).

To encode the length of day, we modulated the amplitude envelope of the filtered noise sinusoidally between zero amplitude and full amplitude. The frequency of this modulation was linearly proportional to the length of the day. The analogy of this strategy was the cycle of sunrise and sunset on a planet: a day would be perceived as a gradual increase in lightness from the sun (increasing volume), and decay with the end of the day (decreasing volume). The planets vary tremendously in speed of rotation around their axis. However, a constant scaling factor was applied that allowed most planets to fall in the range of human rhythm cognition [26], leaving only a few planets (e.g., Mercury, Venus), whose length of day were too long for the percept of a tactus (recognizable beat) to form. The transformation we used converted 24 hours ( 1 day on earth) into 1 second.

The length of year was conveyed using spatial location and speed of revolution around the listener. The shorter the length of year, the faster it would revolve around the listener. Due to the tremendous range of the length of years in the planets, these were conveyed in two phases. One phase for the four terrestrial planets, which have shorter years, and a second for the four gas giants, which have longer years. Jupiter was used as a reference to link 
between the two phases. In the first phase, it moved the slowest. In the second phase, it moved the fastest.

To convey distance from the sun, a spaceship traveled to each planet in sequence starting at the Sun and finishing on Neptune. Although there is no sound in space, a fictitious sound effect was created for the spacecraft with additional sound effects for passing objects like asteroids. This base sound was used to convey the distance between the first four terrestrial planets, which would be 3-4 seconds apart. To get to the gas giants, the ship was "accelerated" to five times its normal speed to reach Jupiter and Saturn, and accelerated to ten times the normal speed to reach Uranus and Neptune. The acceleration and velocity were conveyed by increasing the playback speed of the spaceship sound proportionally to the new speed, meaning a faster spaceship speed had a higher pitch. This acceleration had the affect of allowing the ship to reach the planets within a reasonable time-scale in the show.

\subsection{Planetary View}

Based on our interviews with astronomy educators, we chose specific features to cover for each of the planets: moons, rings, temperature range, gravitational strength, and type of planet. We mapped the number of moons to an equal number of highfrequency sinusoids rotating at variable speeds around the listener. The frequency of each moon was within a 3 octave range above $\mathrm{C} 5$ and varied in loudness, but unlike in the solar system view, the precise mass and rotational period of each moon was not used in the mapping. Frequencies were randomly selected with equal probability within the range. Using sinusoids instead of brown-noise gave the moons a bell-like sound and also increased the differentiability of each moon. This choice allowed more moons to be conveyed in a narrower high-frequency space than if we had used filtered noise, gracefully handling the difference between representing just Earth's moon versus over 60 belonging to Jupiter and Saturn.

We encoded the number of rings through a distribution of variable pitch and loudness pure-tones as well, but instead of having specific spatial locations, they were played with equal amplitude through all the speakers. Additionally, a larger frequency range was used (extending downwards to $\mathrm{C} 3$ ) meaning that a ring could be conveyed using a much lower pure tone than a moon. Each pure-tone represented one ring, making scalability of number of rings easily portrayed.

An auditory graphing approach was used to display the temperature range of each planet whereby cold temperature was conveyed by low pitch and the highest temperature was conveyed by high pitch. Temperatures were first normalized across the planets and then the mean normalized temperature was multiplied by 1500 $\mathrm{Hz}$ with $200 \mathrm{~Hz}$ added to each. The pitch range was determined by multiplying the normalized temperature range of each planet by $1500 \mathrm{~Hz}$. Short, $10 \mathrm{~Hz}$ sine-grains were used to convey the temperature progression, which would increase and decrease at a rate proportional to the length of day, conveying that the temperature was a function of length of day.

The effect of gravity is proportional to the mass of each planet, and another sonification approach was used to convey the magnitude of the force of gravity. Using a physical model of a bouncing object, the effect of gravity was manipulated according to the force of gravity on the planet. As with the original mass mapping, the pitch of the sound of the ball hitting the surface was proportional to the size of the planet, so larger planets had a lower pitch than smaller planets.

The surface characteristics of the planets can be categorized into two broad types: terrestrial and gas giant. We designed a continuous timbre space to convey each type, which depended upon planetary mass and density. Beginning with the previously discussed mass-dependent fundamental frequency, a variable number of harmonics were added, the number of which increased logarithmically with mass. To convey density, the sound was passed through a low-pass filter whose cutoff frequency decreased with increasing density. This design gave the more diffuse gas giants a lower but richer timbre than the terrestrial planets, who were higher in pitch and more mellow in timbre.

\subsection{Script Description}

Although sonification was used to convey the information of the planets, speech was used to provide a sense of narrative to the show, explain to the audience what was being heard, and provide additional context. The script was written and recorded in a sound-isolated recording room and included two male narrators. The script had four sections: an introduction, the Solar System View, the Planetary View, and a conclusion. The first three portions of the show were used to present the different mappings and various facts about the planets, and the show concluded with an independent musical composition chosen for the show.

\section{PLANETARIUM INSTALLATION}

\subsection{Location and Setup}

After completing the design of the auditory display mappings, recordings, and the accompanying speech descriptions, we presented the show in a local planetarium at the end of April 2016. This event was free and open to the public. Participants were informed about the show through emails to campus mailing lists, posters, and advertisements posted at the science center in the weeks up to the show. While there is some potential for recruitment bias, the attendees (individuals who go out of their way to attend an ILE experience on a weeknight) are actually very representative of a typical evening show attendee.

The show leveraged the planetariums' quadraphonic speaker system for the spatial audio mappings, and prior to the show the gain of each speaker was referenced across frequencies to check for anomalies in the frequency response. As a way to supplement the sonifications and the descriptive script for the show, we created a PowerPoint presentation which contained images of each planet to be projected onto the dome as visual anchors for each of the sonifications. Using the four built-in projectors for the planetarium, this slide show accompanied the sonification.

As attendees entered the planetarium, we informed them about the exploratory nature of this research and asked if they wanted to provide feedback through a short paper survey at two points during the show. For attendees who agreed, they were given the survey that was filled out on two occasions, as follows.

For the first part of the show, attendees listened to a brief introduction and then the Solar System View, which included sonifications for the mass of each planet, the length of day, length of year, and distance from the sun. These were presented in groups, starting with Mercury and moving out toward Neptune (i.e., the mass for each planet was played, then the length of day, etc.). After the first part was completed, we paused the show and asked the 
audience to complete a short survey of five six-point Likert-type questions regarding information mappings from the past series of sounds. As the audience was not able to individually explore the sounds at their own pace, we did not ask about individual sound mappings for each planet, but instead focused on the overall coherency and understandability of the sounds as a whole.

After the break for the first half of the survey, we resumed the second portion of the show. It contained two parts: the Planetary View and the conclusion. The Planetary View included separate sections for each planet's number of moons, presence (and number) of rings, overall temperature range, type of planet, and a representation of gravitational strength. Using sonifications in the planetary view allowed us to make comparisons which someone might not be able to easily make comparing static images of the planets. One example is how Venus and Uranus have very similar gravitational strength, though just observing them would not lead to this conclusion. During the show, their gravities (represented by the ball bouncing metaphor) were sequentially played making them easier to compare through other means. Similar comparisons were made between Uranus and Neptune, which have very similar characteristics (temperature and composition).

The final portion of the show was an artistic composition that recalled different auditory displays from the earlier, introductory portions of the show. At the end of the performance, the attendees completed the second set of Likert-type questions (about the planetary view mappings) and a series of free response questions regarding their overall listening experience.

\subsection{Survey Description}

The survey included two sections of Likert-type questions (one set for the Solar System View and one for the Planetary View), where the attendees were asked to rate the sounds on their aesthetics, helpfulness, and relatability to astronomical concepts:

1. How interesting were the sounds?

2. How pleasant were the sounds?

3. How helpful were the sounds?

4. How easy was it to understand the sounds?

5. How relatable were the sounds to their ideas?

We chose to use a six-point scale instead of a more typical five or seven-point scale to let the attendees give a range of either positive or negative feedback. Measuring the user experience for a listener can be difficult and sound often evokes diverse feelings from all individuals. The even scale was used to encourage the audience to be honest about their opinions through introspective reflection instead of choosing the (possibly) easier neutral option. For each question, one represented the lowest rating and six represented the highest.

The free response questions asked about overall likes and dislikes of the show, and asked about the attendee's favorite sound or set of sounds. We explored how well the audience members thought the planets were represented based on their previous knowledge. We asked the audience about how the sonifications made them feel, and whether or not they learned something interesting about the solar system or now appreciate another detail they did not know previously. Finally, we asked them if any portion of their understanding of the solar system has changed.

We asked two demographics questions, as the audience was open to all members of the community: age and student status (if a student, we also asked for current year in school).

\section{RESULTS}

Over 50 attendees came the night of the planetarium show, and 40 people completed the survey providing feedback about their experience throughout and after the show. Those who completed the survey reported their ages to be between 11 and 63, with the majority being in their 20s. Nineteen reported being students (ranging from fifth grade through graduate school). The attendees were asked to rate the sonifications to give high-level feedback on the sounds for the Solar System View and the Planetary View. As this was an initial exploration of a sonification model of the solar system, we did not perform inferential statistical analyses, but instead focused on descriptive statistics such as average ratings and the standard deviations (SD) of those ratings.

\subsection{Quantitative Feedback}

During the show, attendees responded to two categories of questions regarding the aesthetics (interesting and pleasant) and usefulness (helpful, understandable, and relatable) of the sonifications for the Solar System and Planetary Views. Table 1 presents the average rating for each question, while the detailed distributions can be found in Figure 1, Figure 2, and Figure 3. Each of the five categories achieved high ratings (at least 4.3 out of 6 ). The audience rated the aesthetics of the sounds especially highly (at least a 4.7 out of 6), with one category (Planetary View-interesting) having a 5 out of 6 . Our carefully designed model of the solar system and information mappings was found tasteful and fitting by the attendees.

\begin{tabular}{llclc}
\hline \multicolumn{1}{c}{ Criteria } & \multicolumn{2}{c}{ Solar System View } & \multicolumn{2}{c}{ Planetary View } \\
\hline Interesting? & 4.83 & $(1.11)$ & 5.00 & $(0.95)$ \\
Pleasant? & 4.73 & $(0.96)$ & 4.84 & $(0.92)$ \\
Helpful? & 4.68 & $(0.73)$ & 4.55 & $(0.89)$ \\
Understandable? & 4.75 & $(0.90)$ & 4.31 & $(0.86)$ \\
Relatable? & 4.59 & $(0.91)$ & 4.49 & $(1.02)$ \\
\hline
\end{tabular}

Table 1: On a scale of 1 to 6 , the users gave average ratings listed (and standard deviations).

The second grouping of questions sought to measure the audience's perceived usefulness of the auditory display and the overall understandability of the sonification mappings. Overall, the audience rated these three categories for both views quite highly, though the Solar System View had slightly higher scores. Ease of understanding for the Planetary View proved most difficult for the audience, perhaps due to each planet containing up to six different sonifications; across eight planets, this could lead to some confusion.

The most agreement between ratings (i.e., the responses that had the lowest variability) were for the helpfulness of the sonifications in understanding the concepts presented for both the Solar System and Planetary Views. The qualitative feedback from the audience in the next section provides some examples of how the sonifications were helpful for increasing their understanding of our solar system. 


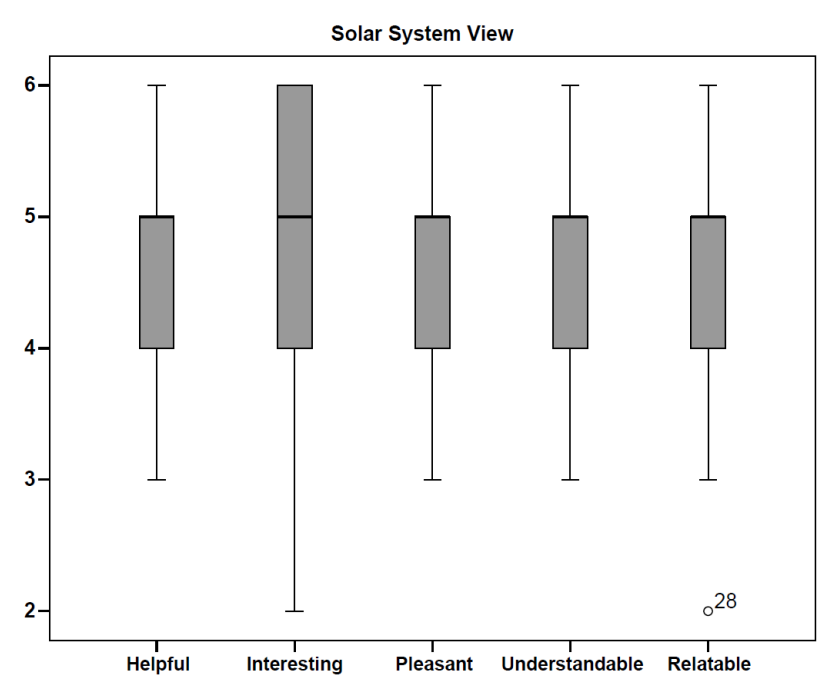

Figure 1: A series of boxplots representing the variation of the Solar System View Likert responses.

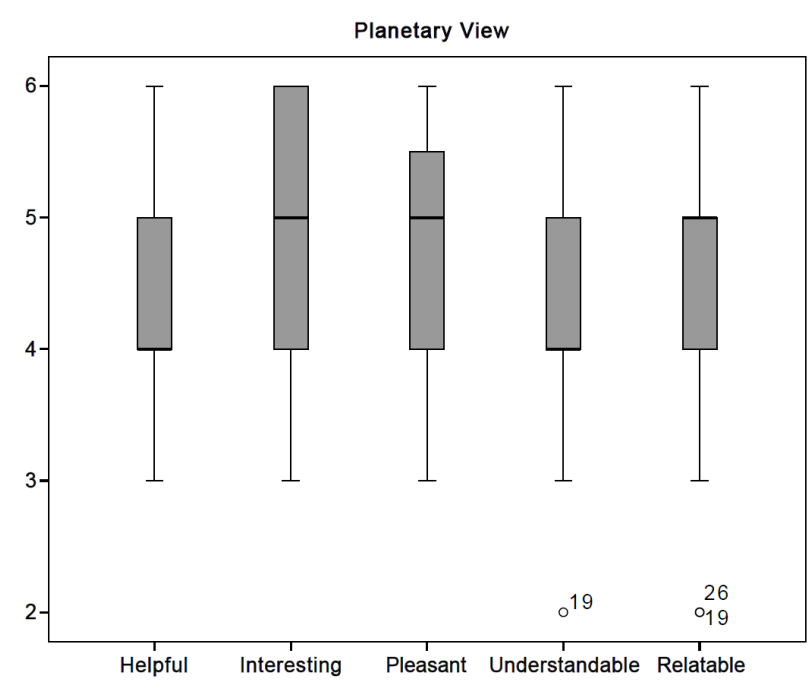

Figure 2: A series of boxplots representing the variation of the Planetary View Likert responses.

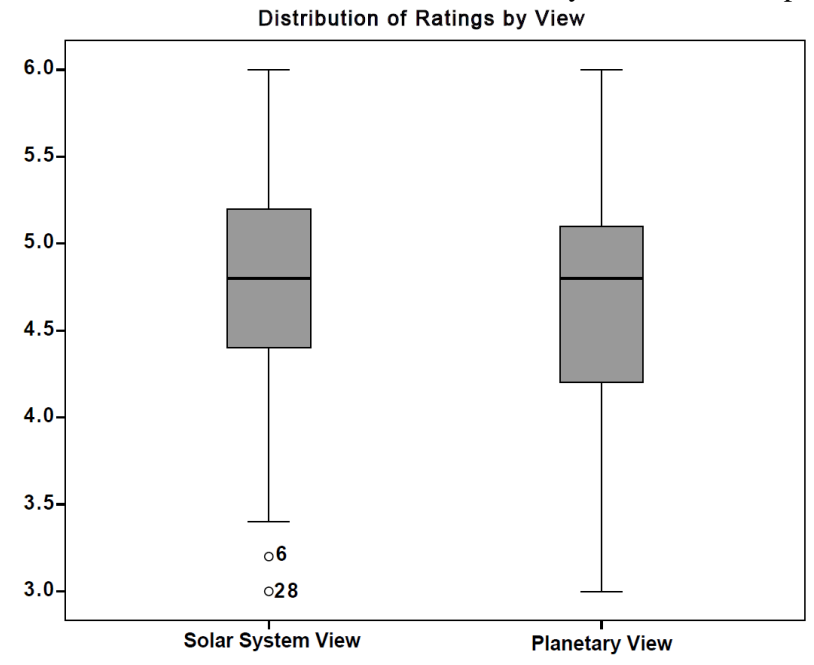

Figure 3: Two boxplots representing the overall variation range for both views.

\subsection{Qualitative Feedback}

In addition to the Likert-type ratings, we asked free response questions at the end of the show. These questions included listener likes or dislikes, favorite sounds, if there was something new they learned, and if they had any affect (emotional reaction) when listening to the solar system sonification. Many of the attendees reported particularly enjoying the sounds for the gas giants, the sounds of planets orbiting around them, the mapping for gravity (the ball bounce model), and they really enjoyed the portions that compared different planets to each other across one or two variables such as temperature, size, and distance from the sun.

Some of the respondents reported that it was much harder to remember the sounds during the second portion of the show, where we individually introduced information about each of the planets. There were many more details to remember in this section than in the first portion, which might explain the slightly lower aver- ages in the second half of the show. However, even with those ratings, many people stated that they better understood the scale and the relationship between the planets; these comparisons were presented in the planetary view. Eighteen of the 40 survey respondents $(45 \%)$ listed at least one piece of information they learned during the show that they had not known before, with those responses being evenly split between the first and the second half.

When asked if their understanding of the solar system changed (and how), one attendee reported, "Yes, the relationship between the planets is clearer" and another mentioned that they "realized the vast differences [between the planets]" after the show. Ten respondents provided comments similar to one attendee, who remarked that "The sound really helped put the distance in perspective," referring to the first half of the show, and that "relative differences were very evident and cool to listen to." During the interviews at the beginning of this research, the astronomy instructors mentioned one of the hardest concepts for students to understand 
is the size and scale of the solar system. The sonifications helped the audience to understand them in a way they had not previously.

Many of the attendees recognized comparisons they had not previously noticed such as how the "weather/atmosphere comparisons with rings and moons was really interesting and informative." When respondents listed the details about the solar system they learned during the planetarium show, they reported that they had a better understanding of the relationships and comparisons between planets, especially gravity.

Finally, we asked all of the attendees to provide feedback about their overall experience during the show and we prompted them to explain how listening to the planets made them feel. Nineteen out of the 27 respondents who answered this question (70\%) specifically stated how happy, joyful, or otherwise positive they felt from listening to the sonifications. One of the attendees explained how their mood changed throughout the show:

At first it was really overwhelming, almost headache inducing; however, the more the sounds were explained and pulled apart, the more meaningful and enjoyable it became.

A few individuals reported how they felt "unexpectedly" relaxed while listening to the sonifications, and many reported how "pleasant and interesting" the experience was. For others, the sounds triggered strong aesthetic experiences, reporting that it made them feel "small and insignificant," while others more positively reflected that the sounds made them feel "awesome and ethereal."

There were no real differences between the ratings from the students based on grade, though the students in the lowest grades gave lower overall ratings to the five criteria than the older students did. Additionally, those attendees who did not mention deeper understanding of the solar system or learning new information had much lower ratings for the sonifications than those who did. Exploring these lower ratings more in-depth would be important before future deployment of the solar system sonifications in any other informal or formal learning context.

\section{DISCUSSION}

Overall, the audience gave each of the sonifications high ratings on average, with the highest scores from respondents on the aesthetic design of the solar system model. The well-designed sonifications provided an enjoyable experience to all audience members. Understanding and relating the sounds to their ideas proved most complex for the audience, and using their feedback to iterate on the model will decrease complexity and increase usefulness in future deployments.

The Planetary View presented many more individual details, and the attendees reported having more trouble remembering the sonifications for each of them. A way to support better learning and understanding of those portions of the sonification could be through an interactive exhibit, where the user could individually control and explore the sounds at their own pace. For future planetarium shows, we might also select a few representative cases for comparisons instead of presenting each interesting comparison available. Another possibility includes using smaller sections for each data characteristic instead of combining characteristics for one planet into a single section (presenting gravity, temperature, etc. in groups instead of every detail for one planet then the next).

Future work should also include evaluating the sounds' abilities to support learning this content directly. The evaluation for this show focused on exploring the audience's reaction and their experience attending a multi-modal ILE sonification. Additional deployments using the sonification as a show experience for student field trips would fit with the other major use case of the planetarium, and would present an opportunity to evaluate the sonification model in a way which might reduce potential confounds from demand characteristics (i.e., the students might be asked to give feedback on the show from the normal planetarium instructor, and not have biases from the researcher's introduction).

\section{CONCLUSION}

Planetariums typically rely on visuals (with varying levels of speech descriptions), but have not explored using sonifications or other auditory displays as a way to present information about space. Our positive results with respect to helpfulness, interest, pleasantness, understandability, and relatability of concepts demonstrate that attendees at a planetarium show can enjoy and learn information about space and our solar system by listening. In particular, the mappings and sound design used in our model of the solar system were successful, and future applications could involve a much wider audience, including visitors with vision impairment. Further study of the sonifications in a more traditional classroom context could also provide an interesting way to engage a diverse group of students through a multi-modal experience.

\section{ACKNOWLEDGMENTS}

Portions of this work were supported by funding from the National Science Foundation (NSF) and from the National Institute on Disability, Independent Living, and Rehabilitation Research (NIDILRR). Thank you to the local science center for supporting and encouraging this work.

\section{REFERENCES}

[1] S. Allen, "Designs for learning: Studying science museum exhibits that do more than entertain," Science Education, vol. 88, no. S1, pp. 17-33, 2004, http://doi.org/10.1002/sce. 20016.

[2] M. S. Horn, E. T. Solovey, R. J. Crouser, and R. J. Jacob, "Comparing the use of tangible and graphical programming languages for informal science education," in Proceedings of the SIGCHI Conference on Human Factors in Computing Systems. ACM, 2009, pp. 975-984, http://dl.acm.org/ citation.cfm?id=1518851.

[3] B. N. Walker, M. T. Godfrey, J. E. Orlosky, C. Bruce, and J. Sanford, "Aquarium sonification: Soundscapes for accessible dynamic informal learning environments." 12th International Conference on Auditory Display, ICAD 2006, 2006, https://smartech.gatech.edu/handle/1853/50426.

[4] Z. Obrenovic, J. Abascal, and D. Starcevic, "Universal accessibility as a multimodal design issue," Communications of the ACM, vol. 50, no. 5, pp. 83-88, 2007, http://www.realtechsupport.org/UB/I2C/ UniversalAccessibility_2007.pdf.

[5] A. Elliott, "Developing accessible museum curriculum: the research, development and validation of a handbook 
for museum professionals and educators," Ph.D. dissertation, Kansas State University, 2007, http://krex.k-state.edu/ dspace/handle/2097/295.

[6] R. E. Grinter, P. M. Aoki, M. H. Szymanski, J. D. Thornton, A. Woodruff, and A. Hurst, "Revisiting the visit:: understanding how technology can shape the museum visit," in Proceedings of the 2002 ACM conference on Computer supported cooperative work. ACM, 2002, pp. 146-155, http://dl.acm.org/citation.cfm?id=587100.

[7] G. Anagnostakis, M. Antoniou, E. Kardamitsi, T. Sachinidis, P. Koutsabasis, M. Stavrakis, S. Vosinakis, and D. Zissis, "Accessible museum collections for the visually impaired: combining tactile exploration, audio descriptions and mobile gestures," in Proceedings of the 18th International Conference on Human-Computer Interaction with Mobile Devices and Services Adjunct. ACM, 2016, pp. 1021-1025, http://doi.org/10.1145/2957265.2963118.

[8] A. Dulyan and E. Edmonds, "Auxie: initial evaluation of a blind-accessible virtual museum tour," in Proceedings of the 22nd Conference of the Computer-Human Interaction Special Interest Group of Australia on Computer-Human Interaction. ACM, 2010, pp. 272-275, http://dl.acm.org/citation. cfm?id=1952280.

[9] M. Jeon, R. J. Winton, J.-B. Yim, C. M. Bruce, and B. N. Walker, "Aquarium fugue: interactive sonification for children and visually impaired audience in informal learning environments." 18th International Conference on Auditory Display, ICAD 2012, 2012, https://smartech.gatech.edu/ handle/1853/44427.

[10] B. N. Walker and M. A. Nees, "Theory of sonification," in The sonification handbook, A. H. Thomas Hermann and J. G. Neuhoff, Eds. Berlin: Logos Publishing House, 2011, pp. 9-39.

[11] J. C. Ballesteros and B. Luque Serrano, "Using sounds and sonifications for astronomy outreach," 2008, http://oa.upm. es/4724/.

[12] H. Harger and A. Hyde, "Broadcasting the music of the spheres: Creating radio astronomy," in 55th International Astronautical Congress, 2004, http://doi.org/10.2514/ 6.IAC-04-IAA.6.16.1.04.

[13] P. Lunn and A. Hunt, "Listening to the invisible: Sonification as a tool for astronomical discovery," 2011, http: //eprints.hud.ac.uk/15922.

[14] E. Landi, R. Alexander, J. Gruesbeck, J. Gilbert, S. T. Lepri, W. Manchester, and T. H. Zurbuchen, "Carbon ionization stages as a diagnostic of the solar wind," The Astrophysical Journal, vol. 744, no. 2, p. 100, 2011, http://dx.doi.org/10. 1088/0004-637X/744/2/100.

[15] M. Ballora, "Sonification strategies for the film rhythms of the universe." 20th International Conference on Auditory Display, ICAD 2014, 2014, https://smartech.gatech.edu/ handle/1853/52075.

[16] M. Quinton, I. McGregor, and D. Benyon, "Sonifying the solar system," 2016, http://www.icad.org/icad2016/ proceedings2/papers/ICAD2016_paper_3.pdf.

[17] J. McCartney, "Rethinking the computer music language: Supercollider," Computer Music Journal, vol. 26, no. 4, pp. 61-68, 2002.
[18] S. Wilson, D. Cottle, and N. Collins, The SuperCollider Book. The MIT Press, 2011.

[19] G. Dubus and R. Bresin, "A systematic review of mapping strategies for the sonification of physical quantities," PloS one, vol. 8, no. 12, p. e82491, 2013.

[20] D. R. Williams, "Planetary fact sheet," 2015, [Online; accessed 18-February-2017]. [Online]. Available: http: //nssdc.gsfc.nasa.gov/planetary/factsheet/

[21] A. S. Bregman, "Auditory scene analysis: Hearing in complex environments," in Thinking in sound: The cognitive psychology of human audition. Cambridge: MIT Press, 1993, pp. 10-36.

[22] G. Kramer, "Some organizing principles for representing data with sound," in Santa Fe Institute Studies in the Science of Complexity - Proceedings Volume, vol. 18. AddisonWesley Publishing Co, 1994, pp. 185-185.

[23] J. H. Schuett and B. N. Walker, "Measuring comprehension in sonification tasks that have multiple data streams," in Proceedings of the 8th Audio Mostly Conference. ACM, 2013, p. 11, http://doi.org/10.1145/2544114.2544121.

[24] J. H. Schuett, R. J. Winton, J. M. Batterman, and B. N. Walker, "Auditory weather reports: demonstrating listener comprehension of five concurrent variables," in Proceedings of the 9th Audio Mostly: A Conference on Interaction With Sound. ACM, 2014, p. 17, http://doi.org/10.1145/2636879. 2636898.

[25] B. N. Walker, "Magnitude estimation of conceptual data dimensions for use in sonification," Journal of Experimental Psychology, vol. 8, no. 4, pp. 211-221, 2002, http://doi.org/ 10.1037/1076-898X.8.4.211.

[26] C. L. Krumhansl, "Rhythm and pitch in music cognition." Psychological bulletin, vol. 126, no. 1, p. 159, 2000. 